\title{
Inyección intratecal inadvertida de ácido tranexámico
}

Inadverted intrathecal injection of tranexamic acid Injeção intratecal inadvertida de ácido tranexâmico

\section{Dr. Santiago Ayala ${ }^{1}$}

\section{Resumen}

Se realiza una revisión descriptiva sobre la inyección de ácido tranexámico en el espacio subaracnoideo. Se destaca que un error puede tener consecuencias catastróficas sobre el paciente, con un alto porcentaje de mortalidad. Se analizan las posibles causas que pueden llevar a la inyección errónea. Se advierte sobre la existencia de preparaciones de ácido tranexámico de similar apariencia a las de la bupivacaína de uso intratecal. Se describe el cuadro clínico de la complicación, el mecanismo de toxicidad, los tratamientos utilizados, y la evolución de los casos relatados en las referencias encontradas. Se discuten estrategias para evitar la complicación, señalando que la seguridad no debe basarse en la perfección humana, sino en medidas que dificulten cometer errores.

Palabras clave: Ácido tranexámico Inyección intratecal

Key words: $\quad$ Tranexamic acid Intrathecal injection

\section{Introducción}

La inyección errónea de un fármaco en la práctica anestésica es una lamentable eventualidad a la que está expuesto todo anestesiólogo.

El ácido tranexámico (ATX) es un antifibrinolítico usado para disminuir el sangrado quirúrgico. Si es inyectado en forma inadvertida en el espacio subaracnoideo genera graves complicaciones y en ocasiones la muerte.

Esa nefasta posibilidad se ve favorecida por la existencia de formulaciones de ATX con una presentación similar a la de fármacos anestésicos locales de uso intrarraquídeo.

El propósito de este trabajo es realizar una revisión descriptiva de los casos reportados, centrando la atención en el cuadro clínico resultante, el mecanismo de acción, las posibles causas del error, el manejo de la complicación, su posible evolución, y, a la vez, establecer recomendaciones para prevenir el error.

\section{Material y método}

Se realizó una búsqueda en la National Library of Medicine's PubMed Database y en Google Scholar, en idioma inglés, desde enero de 2010 a junio de 2020, utilizando como palabras clave tranexamic acid e intrathecal injection. Se analizaron los artículos resultantes, así como las referencias señaladas que se consideraron relevantes.

\section{Desarrollo}

Se encontraron 21 reportes de casos clínicos de inyección intratecal de ATX que resultaron de publicaciones y sus referencias bibliográficas ${ }^{(1-21)}$ (tabla 1), y de artículos de revisión en el período analizado ${ }^{(22)}$.

Todos los casos reportados fueron por confusión por un similar aspecto de las ampollas de ATX con ampollas

1. Profesor Adjunto de Anestesiología. Cátedra de Anestesiología. Facultad de Medicina. Universidad de la República, Uruguay. Fellow of Interventional Pain Practice (FIPP). World Institute of Pain (WIP).

El autor declara no tener conflictos de intereses.

Correspondencia: Dr. Santiago Ayala. Correo electrónico: ayalapastorino@gmail.com

Recibido: $17 / 8 / 2020$

Aprobado: 21/10/2020

Attribution-NonCommercial 4.0 International (CC BY-NC 4.0) 
Tabla 1. Detalle de los casos publicados.

\begin{tabular}{|c|c|c|c|}
\hline Autor & Cirugía & Dosis ATX & Evolución \\
\hline Wong $^{(1)}$ & Apéndice & $75 \mathrm{mg}$ & $\begin{array}{l}\text { Recuperación total, } \\
24 \text { horas }\end{array}$ \\
\hline De Leede ${ }^{(2)}$ & RTU & $50 \mathrm{mg}$ & Recuperación, 1 mes \\
\hline$Y_{e h}^{(3)^{*}}$ & Colon & $500 \mathrm{mg}$ & $\begin{array}{l}\text { Fallece. Fibrilación } \\
\text { ventricular }\end{array}$ \\
\hline Garcha $^{(4)}$ & Fract. tibia & $300 \mathrm{mg}$ & $\begin{array}{l}\text { Fallece. Fibrilación } \\
\text { ventricular }\end{array}$ \\
\hline Mohseni $^{(5)}$ & Fract. rodilla & $350 \mathrm{mg}$ & $\begin{array}{l}\text { Recuperación total, } \\
6^{\circ} \text { día }\end{array}$ \\
\hline Sabzi(i) & Cesárea & $500 \mathrm{mg}$ & $\begin{array}{l}\text { Fallece. Fibrilación } \\
\text { ventricular }\end{array}$ \\
\hline $\mathrm{Kim}^{(7)}$ & Fract. tobillo & $220 \mathrm{mg}$ & $\begin{array}{l}\text { Fallece. Paro } \\
\text { cardíaco }\end{array}$ \\
\hline Veisi ${ }^{(8)}$ & Cesárea & $500 \mathrm{mg}$ & $\begin{array}{l}\text { Fallece. Fibrilación } \\
\text { ventricular }\end{array}$ \\
\hline Kaabachi ${ }^{(9)}$ & Atroscopía & $90 \mathrm{mg}$ & $\begin{array}{l}\text { Recuperación total, } \\
4^{\circ} \text { día }\end{array}$ \\
\hline Srivistava $^{(10)}$ & Colecistectomía & $350 \mathrm{mg}$ & $\begin{array}{l}\text { Fallece. Paro } \\
\text { cardíaco }\end{array}$ \\
\hline Mahmoud ${ }^{(11)}$ & Miembro inferior & $300 \mathrm{mg}$ & $\begin{array}{l}\text { Recuperación total, } \\
24 \text { horas }\end{array}$ \\
\hline Butala $a^{(12)^{*}}$ & Litotricia & $300 \mathrm{mg}$ & $\begin{array}{l}\text { Recuperación total, } \\
5^{\circ} \text { día }\end{array}$ \\
\hline Raghu $^{(13)}$ & Cesárea & $250 \mathrm{mg}$ & $\begin{array}{l}\text { Fallece. Arritmia } \\
\text { ventricular }\end{array}$ \\
\hline Antwi ${ }^{: 14}$ & Cesárea & $200 \mathrm{mg}$ & $\begin{array}{l}\text { Fallece. Fibrilación } \\
\text { ventricular }\end{array}$ \\
\hline Goyal ${ }^{(15)}$ & Hernia & $250 \mathrm{mg}$ & $\begin{array}{l}\text { Recuperación total, } \\
7^{0} \text { día }\end{array}$ \\
\hline $\operatorname{Roy}^{(16)}$ & Cesárea & $200 \mathrm{mg}$ & $\begin{array}{l}\text { Recuperación total, } \\
3^{\text {er }} \text { día }\end{array}$ \\
\hline $\operatorname{Narra}^{(17)^{* *}}$ & Próstata & $250 \mathrm{mg}$ & $\begin{array}{l}\text { Recuperación total, } \\
6^{\circ} \text { día }\end{array}$ \\
\hline $\operatorname{Hatch}^{(18)}$ & Cesárea & $200 \mathrm{mg}$ & $\begin{array}{l}\text { Fallece. Falla del } \\
\text { ventilador }\end{array}$ \\
\hline Elkhateeb $^{(19)}$ & Cesárea & $160 \mathrm{mg}$ & $\begin{array}{l}\text { Fallece. Fibrilación } \\
\text { ventricular }\end{array}$ \\
\hline Grissinger $r^{(20)}$ & Rodilla & - & $\begin{array}{l}\text { Alta, } 23^{\text {er }} \text { día. } \\
\text { Rehabilitación }\end{array}$ \\
\hline Med. Errors ${ }^{(21)}$ & Cadera & - & Alta. Rehabilitación \\
\hline
\end{tabular}

de bupivacaína $0,5 \%$ hiperbárica de uso intrarraquídeo, salvo en un caso en el que el ATX fue inyectado a través de un catéter subaracnoideo colocado para administrar morfina ${ }^{(3)}$.

El cuadro clínico se acompañó de una falla del bloqueo sensorial y motor, y se manifestó inicialmente por dolor lumbar, tetania con hiperextensión lumbar y sacudidas de los miembros inferiores, seguidas de convulsiones generalizadas, inestabilidad hemodinámica con hipertensión o hipotensión, pérdida de la conciencia, taquicardia ventricular, fibrilación ventricular refractaria o paro cardíaco con colapso cardiovascular. Los síntomas se instalaron, en general, inmediatamente luego de la inyección, salvo en un caso en el que se manifestaron a las cuatro horas ${ }^{(1)}$, o al revertir la anestesia general en aquellos casos en los que se la aplicó ante el fracaso de la anestesia regional.

En todos los casos el tratamiento consistió en un rápido soporte cardiopulmonar y la administración, según el caso, de fenitoína, tiopental, diazepam, relajantes musculares y antiarrítmicos. En dos casos se realizó lavado del líquido cefalorraquídeo (LCR) ${ }^{(12,17)}$.

En cuanto a la evolución, de los 21 reportes encontrados, diez fallecieron, siete por fibrilación ventricur $\operatorname{lar}^{(3,4,6,8,13,14,19)}$, dos por paro cardíaco con colapso cardiovascular ${ }^{(7,10)}$, y uno por falla del ventilador ${ }^{(18)}$. De los 11 pacientes que sobrevivieron, la mayoría lo hizo prácticamente sin secuelas ${ }^{(1,2,5,9,11,15-17,20)}$, salvo un caso que quedó con una paresia peroneal bilateral ${ }^{(2)}$, y otros dos casos que requirieron rehabilitación prolongada ${ }^{(20,21)}$. Los dos pacientes en los que se realizó el lavado del LCR sobrevivieron $^{(12,17)}$.

Del análisis de los 21 casos relatados, sin considerar el caso que falleció por falla del ventilador ${ }^{(18)}$, y los que sobrevivieron pero se desconoce la dosis de ATX recibi$\mathrm{da}^{(20,21)}$, surge que los pacientes que sobrevivieron recibieron dosis de ATX entre 50 y $350 \mathrm{mg}$, con un promedio de $207 \mathrm{mg}$, mientras que los que fallecieron recibieron dosis entre 300 y $500 \mathrm{mg}$, con un promedio de 331 $\mathrm{mg}$. A su vez, en los cuatro pacientes que fallecieron habiendo recibido dosis de $250 \mathrm{mg}$ o menores (sin tener en cuenta el fallecimiento por falla del ventilador), tres correspondieron a cesáreas.

\section{Discusión}

En una encuesta realizada sobre errores cometidos por los anestesiólogos con ampollas de fármacos LASA (Look-alike, Sound-alike, que suenan o parecen iguales), se encontró que el 86,2\% de los encuestados había cometido al menos un error, y que el $66 \%$ de ellos había realizado una inyección equivocada en su carrera ${ }^{(23)}$.

Los accidentes por inyección errónea resultan, en general, de una yuxtaposición de errores, entre los que 


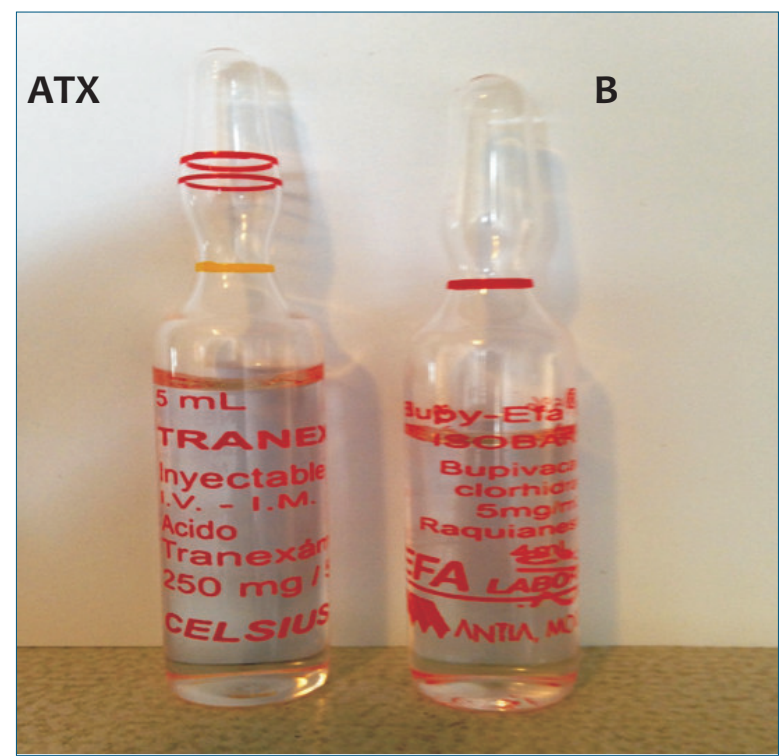

Figura 1. Isoapariencia de las ampollas de ácido tranexámico (ATX) y bupivacaína isobárica (B).

se destaca el generado por la existencia de fármacos LASA $^{(24)}$.

La confusión puede generarse por la similar apariencia de las ampollas por su forma, tamaño, color del cristal, o por el color de las letras serigrafiadas. Por otra parte, esas ampollas generalmente no vienen etiquetadas, sino con el nombre en letra diminuta sobre el vidrio, lo que dificulta su visualización.

Debe señalarse que la presentación de las ampollas depende de la forma en que son comercializadas en los distintos países. De hecho, en Uruguay, la confusión puede darse con la bupivacaína $0,5 \%$ isobárica y no con la bupivacaína al 0,5\% hiperbárica. En la figura 1 se observa la similar apariencia de una preparación de ATX con la de una ampolla de bupivacaína $0,5 \%$ isobárica. Esa similitud resulta aun mayor luego de abiertas, cuando son ofrecidas al anestesiólogo, como puede verse en la figura 2.

Otra posible fuente de error consiste en la devolución equívoca de ampollas de ATX que no han sido usadas en una operación anterior ${ }^{(8)}$. Estas ampollas en lugar de ser devueltas a la farmacia, son ubicadas por error en recipientes que contienen ampollas de bupivacaína con isoapariencia con las de ATX. Debe señalarse que en algunos blocks quirúrgicos las ampollas de ATX son ubicadas en cercanía con las ampollas de uso intrarraquídeo.

Adicionalmente, el posible cansancio del anestesiólogo puede favorecer el equívoco. Es un hecho conocido que los errores vinculados a los fármacos se producen con más frecuencia en el área perioperatoria, donde el

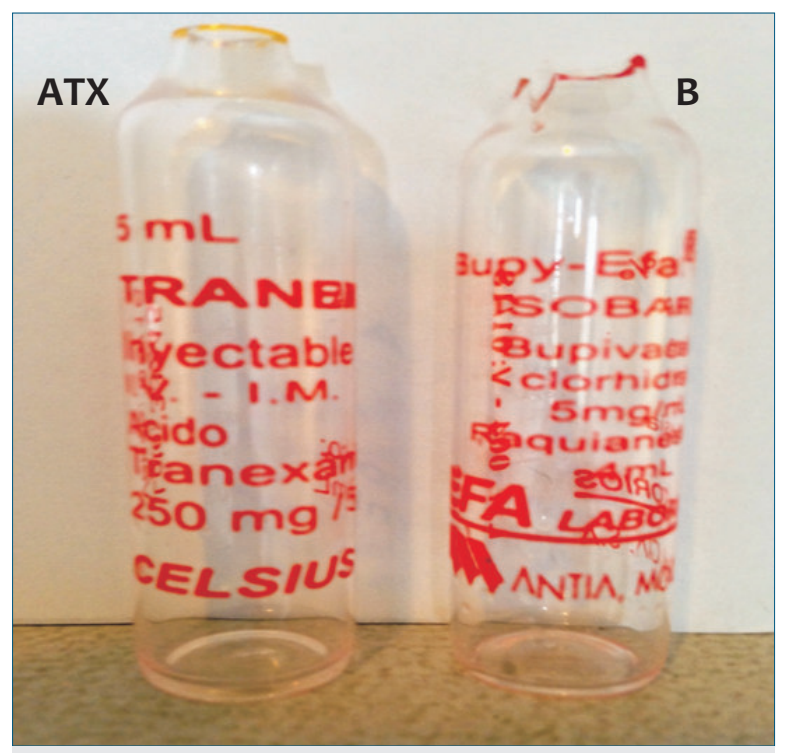

Figura 2. Isoapariencia de las ampollas de ácido tranexámico (ATX) y bupivacaína isobárica (B) luego de abiertas.

estrés juega un papel muy importante ${ }^{(25)}$. La atención del anestesiólogo sobre el paciente, sobre su posición, sobre sus manifestaciones de miedo o dolor, o sobre los registros de los monitores, son factores que pueden actuar como "distractores" a la hora de cargar el fármaco.

En la anestesia regional, en particular, la visualización de la ampolla y la aspiración de su contenido se realizan a una distancia determinada por las condiciones de asepsia al tratarse de ampollas, en general, no estériles, lo que puede facilitar el error.

El ATX es un fármaco antifibrinolítico utilizado para disminuir el sangrado por inhibición competitiva de la activación de plasminógeno a plasmina, por lo que la fibrina no es degradada, resultando en la formación de un coágulo más estable.

Su uso para el control de la hemorragia traumática, quirúrgica y obstétrica, viene en aumento en los últimos años por la evidencia de sus efectos beneficiosos, con reducción de la morbilidad y mortalidad ${ }^{(26)}$.

La Organización Mundial de la Salud la ha incluido entre la lista de medicamentos esenciales, con una fuerte recomendación para su uso precoz en la hemorragia posparto $^{(27)}$.

Si bien es un fármaco disponible en block quirúrgico, no es un fármaco de uso rutinario por los anestesiólogos, quienes muchas veces desconocen su forma de presentación.

Además, los principios básicos en seguridad en lo que tiene que ver con el rótulo de las ampollas, su identificación, o su almacenamiento, no son aplicados con la necesaria rigurosidad por algunas de las instituciones de 
asistencia médica, ni por algunos de los organismos responsables de su aplicación y control ${ }^{(28)}$.

En ese escenario, la seguridad depende exclusivamente de la perfección humana.

El ATX ejerce un efecto epileptógeno cuando es aplicado tópicamente sobre la corteza en animales de experimentación ${ }^{(29-31)}$.

Se sugiere que el ATX intratecal provoca una descarga simpática masiva que resulta en convulsiones, taquicardia, hipertensión y arritmias. Otros autores plantean que las convulsiones son consecuencia de una inhibición de los receptores de ácido gamma aminobutírico (GABA) con bloqueo de su efecto inhibitorio cortical, o como consecuencia de una hipoperfusión cerebral por isquemia vinculada a una vasoconstricción cerebral ${ }^{(18)}$.

La gravedad del cuadro está vinculada a la hiperexcitabilidad nerviosa y a la hiperactividad simpática. El dolor estaría vinculado a la inhibición de los receptores GABA en la espina dorsal ${ }^{(32)}$.

En cuanto al tratamiento, no existe un antídoto para el ATX inyectado intrarraquídeo ${ }^{(10)}$. La base del tratamiento es un rápido soporte cardiopulmonar, la supresión de la actividad convulsiva en base a fenitoína, tiopental o diazepam, y la administración de relajantes musculares y antiarrítmicos.

El lavado del LCR es un procedimiento de emergencia con la finalidad de extraer y diluir el fármaco inyectado, y, por ende, limitar el daño neuronal. Su indicación se basa en reportes clínicos, la mayoría de ellos sobre experiencias con otros fármacos, sobre los que no hay evidencia de utilidad y riesgo, pero es un recurso que puede salvar la vida ${ }^{(33,34)}$.

De hecho, los dos pacientes de la serie que recibieron lavado del LCR sobrevivieron.

Su aplicación dependerá de un balance entre riesgos y potenciales beneficios, de la precocidad con que se hace el diagnóstico, de la estabilidad hemodinámica y del posible control de los movimientos de hiperextensión y convulsivos del paciente.

El volumen total del LCR oscila entre $120 \mathrm{a} 150 \mathrm{ml}$, correspondiendo a un promedio de $34 \mathrm{ml}$, el comprendido entre $\mathrm{T} 12 \mathrm{y} \mathrm{S1}^{(35)}$. En cuanto al volumen que puede ser reemplazado con seguridad, la mayoría de los reportes establecen un volumen de $40 \mathrm{ml}$ en base a experiencias aisladas con distintos fármacos, recomendándose extraer lentamente cantidades de $20 \mathrm{ml}$ por vez, y reemplazarlas por igual cantidad de suero ringer-lactato, suero fisiológico, o una mezcla de ambos, a temperatura corporal $^{(12,33,36)}$.

En relación con las convulsiones, si se mantienen refractarias al tratamiento en base a fenitoína, tiopental o diazepam, se plantea la administración de sulfato de magnesio. Este último se recomienda por su efecto de- presor del sistema nervioso con acción antagonista sobre receptores N-metil-D-aspartato (NMDA) que median en la actividad convulsiva, y también por su acción como inhibidor de la liberación de catecolaminas ${ }^{(28)}$.

Del análisis de los casos analizados, surge que los pacientes que fallecieron recibieron dosis mayores de ATX que los que sobrevivieron.

La elevada mortalidad observada en las cesáreas podría explicarse por una mayor concentración intratecal de ATX en la embarazada, ya que el embarazo reduce el volumen del LCR al disminuir el volumen del espacio subaracnoideo $^{(37)}$.

Es probable que existan casos no reportados y que los publicados representen la punta de un iceberg, considerando que es una complicación que frecuentemente deriva en litigio legal. Para finalizar, debe señalarse que los errores con los fármacos son una inevitable consecuencia de la condición humana, aun entre los profesionales más cuidadosos y responsables ${ }^{(38)}$.

La seguridad no debe basarse en la perfección humana, sino en la aplicación de estrategias que dificulten la concreción del error.

\section{Conclusiones}

Se advierte sobre la existencia de formulaciones de ATX de similar apariencia a las presentaciones de la bupivacaína para uso intrarraquídeo.

Se observa que los casos que fallecieron recibieron dosis de ATX mayores que los que sobrevivieron.

Se señala que no existe un antídoto para esta complicación, planteándose la posibilidad de realizar el lavado del LCR como maniobra de salvataje.

Se enumeran una serie de recomendaciones generales para evitar errores en la administración de fármacos, que resultan particularmente aplicables para el caso del $\operatorname{ATX}^{(37,39-42)}$.

El anestesiólogo deberá realizar un doble chequeo del fármaco previo a su administración, y la etiqueta deberá ser leída cuidadosamente por la enfermera al presentarlo al anestesiólogo y por éste antes de aspirar el contenido.

Se deberá evitar el almacenamiento conjunto de fármacos de similar apariencia o nombre, o el almacenamiento cercano de ampollas de ATX con fármacos de uso intrarraquídeo.

A su vez, se deberá controlar que las ampollas no utilizadas durante las intervenciones sean devueltas a la farmacia, evitando así su retorno al sitio de almacenamiento inicial en block.

Se recomienda informar a los profesionales sobre los fármacos de reciente incorporación y de los errores facilitados por la isoapariencia de las ampollas. 
Los organismos responsables del control sanitario deberán gestionar ante la industria farmacéutica la incorporación de presentaciones de ATX alternativas, y aplicar estrictamente las normas que regulan la identificación de los medicamentos LASA y supervisar su cumplimiento.

\section{Summary}

A descriptive review of tranexamic acid injection in the subarachnoid space is performed. A point is made that this error may have catastrophic consequences on the patient with a high percentage of mortality. Possible causes that can lead to an erroneous injection are analyzed. A warning is made about tranexamic acid preparations being similar in appearance to those of bupivacaine for intrathecal use. The study describes the clinical manifestation of this complication, the toxicity mechanism, treatments used, and the evolution of the cases reported in the references found.

Strategies to avoid complications are discussed, pointing out that safety should not be based on human perfection, but on measures that make it difficult for humans to make mistakes.

\section{Resumo}

Faz-se uma revisão descritiva sobre a injeção de ácido tranexâmico no espaço subaracnóideo. Ressalta-se que é um erro que pode ter consequências catastróficas para o paciente com um elevado percentual de mortalidade. Faz-se uma análise das possíveis causas que podem levar ao uso equivocado de ácido tranexâmico devido a existência de preparações semelhantes em aparência às da bupivacaína para uso intratecal. Descreve-se o quadro clínico da complicação, o mecanismo de toxicidade, os tratamentos utilizados e a evolução dos casos relatados nas referências encontradas.

Discute-se estratégias para evitar complicações, ressaltando que a segurança não deve ser baseada na perfeição humana, mas em medidas que dificultem o erro do ser humano.

\section{Bibliografía}

1. Wong J, Yang S, Tsai M. Accidental injection of tranexamic acid (Transamin) during spinal anesthesia. Ma Zui Xue Za Zhi 1988; 26(2):249-52.

2. de Leede-van der Maarl M, Hilkens P, Bosch F. The epileptogenic effect of tranexamic acid. J Neurol 1999; 246(9):843. doi: 10.1007/s004150050466

3. Yeh H, Lau H, Lin P, Sun W, Mok M. Convulsions and refractory ventricular fibrillation after intrathecal injection of a massive dose of tranexamic acid. Anesthesiology. 2003 Jan;98(1):270-2.
4. Garcha P, Mohan C, Sharma R. Death after an inadvertent intrathecal injection of tranexamic acid. Anesth Analg 2007; 104(1):241-2.

5. Mohseni K, Jafari A, Nobahar M, Arami A. Polymyoclonus seizure resulting from accidental injection of tranexamic acid in spinal anesthesia. Anesth Analg 2009; 108(6):1984-6.

6. Sabzi F, Teimouri H, Zokai A. Myoclonus, seizure and ventricular fibrillation afer intrathecal injection of tranexamic acid. J Tehran Univ Heart Center 2009; 4(4):253-5.

7. Kim M, Jeong S, Choi E, Ha H, Lee H. Death after accidental injection of tranexamic acid during spinal anesthesia. Korean J Leg Med 2009; 33(2):139-42.

8. Veisi F, Salimi B, Mohseni G, Golfam P, Kolyaei A. Accidental intrathecal injection of tranexamic acid in cesarean section: A fatal medication error. Apsf 2010; 25(1):9. Disponible en: https://www.apsf.org/article/accidental-intrathecal-injection-of-tranexamic-acid-in-cesarean-section-a-fatal-medication-error/. [Consulta: 25 agosto2015].

9. Kaabachi O, Eddhif M, Rais K, Zaabar M. Inadvertent intrathecal injection of tranexamic acid. Saudi J Anaesth 2011; 5(1):90-2.

10. Srivastava U, Joshi K, Gupta V, Gupta A, Chauhan N, Dupargude A, et al. Accidental injection of tranexamic acid into subarachnoid space leading to fatal outcome: case report and review. Internet J Anesthesiol 2012; 30(2):1-4.

11. Mahmoud K, Ammar A. Accidental intrathecal injection of tranexamic Acid. Case Rep Anesthesiol 2012; 2012:646028. doi: $10.1155 / 2012 / 646028$

12. Butala B, Shah V, Bhosale G, Shah R. Medication error: Subarachnoid injection of tranexamic acid. Indian J Anaesth 2012; 56(2):168-70.

13. Raghu K, Shrevanni P, Haneef M. Accidental intrathecal injection of tranexamic acid in a term gestation. Int $\mathbf{J}$ Neuro Spinal Sci 2013; 1:1-3.

14. Antw-Kusi A, Sam Awortwi W, Serwaa Hemeng A. Unusual complication following spinal anesthesia for caesarean section. Open J Anesthesiol 2013; 3:275-7. doi: 10.4236/ojanes.2013.35060

15. Goyal G, Vajpayee A, Kant R, Singh R. Refractory status epilepticus after accidental intrathecal injection of tranexamic acid. J Acute Med 2014; 4(2):92-4.

16. Roy A, Sarkar S, BasuThakur S, Kr J. Inadvertent intrathecal administration of tranexamic acid in a case of caesarean section: a report of medication error. SEAJCRR 2015; 4:1910-6.

17. Narra GR. Accidental injection of tranexamic acid into intrathecal space. J Res Anaesthesiol Pain Med 2015; 1:12-4.

18. Hatch D, Atito-Narh E, Herschmiller E, Olufolabi A, Owen M. Refractory status epilepticus after inadvertent intrathecal injection of tranexamic acid treated by magnesium sulfate. Int J Obstet Anesth 2016; 26:71-5.

19. Elkhateeb R, Kamel H. Intrathecal injection of tranexamic acid during caesarean section: accidental fatal mistake. J Clin Obstet Gynecol Infertil 2017; 1(3):1014. 
20. Grissinger M. Key vulnerabilities in the surgical environment: container mix-ups and syringe swaps. P T 2018; 43(3):129-67.

21. Preferred Physicians Medical Risk Retention Group. Medication Errors: intrathecal injection of tranexamic acid and other mix-ups: defending medication errors litigation. Anesth Law 2017; 44:1-6.

22. Patel S, Robertson B, McConachie I. Catastrophic drug errors involving tranexamic acid administered during spinal anaesthesia. Anaesthesia 2019; 74(7):904-14. doi: 10.1111/anae. 14662

23. Rando K, Rey G. Errores de medicamentos LASA en anestesiología en Uruguay. Rev Méd Urug 2017; 33(2):108-25.

24. Ismail S, Taqi A. Medical errors related to look-alike and sound-alike drugs. Anaesth Pain Intensive Care 2013; 17(2):117-22

25. Nanji K, Patel A, Shaikh S, Seger D, Bates D. Evaluation of perioperative medication errors and adverse drug events. Anesthesiology 2016; 124(1):25-34.

26. WOMAN Trial Collaborators. Effect of early tranexamic acid administration on mortality, hysterectomy, and other morbidities in women with post-partum haemorrhage (WOMAN): an international, randomised, double-blind, placebo-controlled trial. Lancet 2017; 389(10084):2105-16. doi: 10.1016/S0140-6736(17)30638-4

27. Roberts I, Kawahara T. Proposal for the inclusion of tranexamic acid (anti- fibrinolytic-lysine analogue) in the WHO model list of essential medicines. Geneva: WHO, 2010.

28. Orser B, U D, Cohen M. Perioperative medication errors: building safer systems. Anesthesiology 2016; 124(1):1-3. doi: 10.1097/ALN.0000000000000905

29. Pellegrini A, Giaretta D, Chemello R, Zanotto L, Testa G. Feline generalized epilepsy induced by tranexamic acid (AMCA). Epilepsia 1982; 23(1):35-45

30. Yamaura A, Nakamura T, Makino H, Hagihara Y. Cerebral complication of antifibrinolytic therapy in the treatment of ruptured intracranial aneurysm. Animal experiment and a review of literature. Eur Neurol 1980; 19(2):77-84.

31. Schlag M, Hopf R, Zifko U, Redl H. Epileptic seizures following cortical application of fibrin sealants containing tranexamic acid in rats. Acta Neurochir (Wien) 2002; 144(1):63-9

32. Ohashi N, Sasaki M, Ohashi M, Kamiya Y, Baba H, Kohno $T$. Tranexamic acid evokes pain by modulating neuronal excitability in the spinal dorsal horn. Sci Rep 2015; 5:13458. doi: $10.1038 /$ srep 13458

33. Liu H, Tariq R, Liu G, Yan H, Kaye A. Inadvertent intrathecal injections and best practice management. Acta Anaesthesiol Scand 2017; 61(1):11-22. doi: 10.1111/aas.12821

34. Tsui B. Common sense medicine and cerebrospinal lavage. Anaesthesia 2014; 69(8):936-7. doi: 10.1111/anae.12796

35. Worris M. Anesthesia del neuroeje. En: Barash P. Anestesia clínica. 8 ed. Barcelona: Wolters Kluwer, 2017:914-44.

36. Tsui B, Malherbe S, Koller J, Aronyk K. Reversal of an unintentional spinal anesthetic by cerebrospinal lavage. Anesth Analg 2004; 98(2):434-6.

37. Patel S, Loveridge R. obstetric neuraxial drug administration errors: a quantitative and qualitative analytical review Anesth Analg 2015; 121(6):1570-7.

38. Jain R, Katiyar S. Drug errors in anaesthesiology. Indian J Anaesth 2009; 53(5):539-42.

39. Jensen L, Merry A, Webster C, Weller J, Larsson L. Evidence-based strategies for preventing drug administration errors during anaesthesia. Anaesthesia 2004; 59(5):493-504.

40. Al-Kadhimi S, Patel A, Plaat F. Intrathecal tranexamic acid - an accident waiting to happen? Int J Obstet Anesth 2018; 34:116-7.

41. Litman R. How to prevent medication errors in the operating room? Take away the human factor. Br J Anaesth 2018; 120(3):438-40.

42. Gupta S, Bhiwal A, Sharma K. Tranexamic acid: beware of anaesthetic misadventures. J Obstet Anaesth Crit Care 2018; $8(1): 1-6$ 